Review

\title{
LncRNAs regulate metabolism in cancer
}

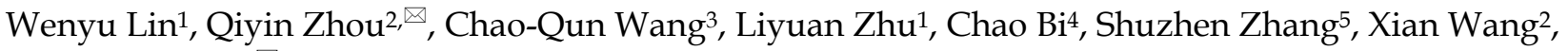 \\ Hongchuan Jin ${ }^{1,}$ \\ 1. Laboratory of Cancer Biology, Key Lab of Biotherapy in Zhejiang, Sir Run Run Shaw Hospital, Zhejiang University School of Medicine, Hangzhou 310016, \\ Zhejiang, China \\ 2. Department of Medical Oncology, Sir Run Run Shaw Hospital, Zhejiang University School of Medicine, Hangzhou 310016, Zhejiang, China \\ 3. Department of Pathology, Affiliated Dongyang Hospital of Wenzhou Medical University, Dongyang 322100, Zhejiang, China \\ 4. Institute of Translational Medicine, Zhejiang University School of Medicine, Hangzhou 310029, Zhejiang, China \\ 5. Department of Obstetrics and Gynecology, Zhejiang Xiaoshan Hospital, Hangzhou 311201, Zhejiang, China \\ $\bowtie$ Corresponding authors: Qiyin Zhou, Ph.D., Email: zhouqiyin@zju.edu.cn. Hongchuan Jin, M.D., Ph.D., Email: jinhc@zju.edu.cn
}

(c) The author(s). This is an open access article distributed under the terms of the Creative Commons Attribution License (https://creativecommons.org/licenses/by/4.0/). See http://ivyspring.com/terms for full terms and conditions.

Received: 2019.09.29; Accepted: 2020.01.18; Published: 2020.02.10

\begin{abstract}
Metabolic reprogramming is a hallmark of cancer. Mammalian genome is characterized by pervasive transcription, generating abundant non-coding RNAs (ncRNAs). Long non-coding RNAs (IncRNAs) are freshly discovered functional ncRNAs exerting extensive regulatory impact through diverse mechanisms. Emerging studies have revealed widespread roles of IncRNAs in the regulation of various cellular activities, including metabolic pathways. In this review, we summarize the latest advances regarding the regulatory roles of IncRNAs in cancer metabolism, particularly their roles in mitochondrial function, glucose, glutamine, and lipid metabolism. Moreover, we discuss the clinical application and challenges of targeting IncRNAs in cancer metabolism. Understanding the complex and special behavior of IncRNAs will allow a better depiction of cancer metabolic networks and permit the development of IncRNA-based clinical therapies by targeting cancer metabolism.
\end{abstract}

Key words: Long non-coding RNA; Cancer; Metabolism

\section{Introduction}

Cancer cells can reprogram metabolism to support them with sufficient building blocks, appropriate redox status and rapid ATP generation in favor of survival, growth or metastasis. For a long time, metabolic reprogramming has been regarded as a hallmark of cancer [1]. Reprogrammed cancer metabolism mainly includes changes of three classes of molecules including carbohydrates, lipids, and amino acids, eventually altering mitochondrial function, glycolysis, glutaminolysis, and lipid metabolism [2]. For example, cancer cells exhibit high rate of aerobic glycolysis, which was known as Warburg effect $[3,4]$. Accumulating evidence indicates that cancer metabolism can be regulated by signaling pathways involved in cell growth and proliferation [5-8]. These studies have expanded our understanding of mechanisms underlying cancer metabolic reprogramming and provided insights into clinical diagnosis, prognosis, and treatment.
However, the multifaceted cancer metabolism still needs further investigations.

Among the genome transcribed, less than $2 \%$ of the genome are protein-coding genes, while more than $90 \%$ of the rest are transcribed into ncRNAs [9]. According to the size, ncRNAs can be subdivided into small ncRNAs such as miRNAs, small nucleolar RNAs (snoRNAs), PIWI-interacting RNAs (piRNAs) and transfer RNA-derived small RNAs (tsRNAs), as well as the newly characterized lncRNA class. LncRNAs are large RNA transcripts longer than 200 nucleotides with no or limited protein-coding potential and limited evolutionary conservation [10]. Among genes expressed in human transcriptome, over $68 \%$ are transcribed into lncRNAs. Based on the locations relative to protein-coding genes, lncRNAs are classified into: (1) sense, or (2) antisense that cover exons of the nearest transcripts in the same or opposite direction; (3) bidirectional, that the initial 
transcription sites of lncRNA and the protein-coding gene on the opposite strand are closely localized, (4) intronic, that the whole IncRNA transcript localizes within the intron of a second transcript, and (5) intergenic, that set within the genomic interval of two genes[11].

LncRNAs have been found to involve in various physiological and pathological cellular activities, such as adipogenesis, inflammation, cell differentiation and tumorigenesis, via genomic expression modulation, epigenetic modification and post-transcriptional regulation in cis or in trans by interacting with chromatins, proteins and RNAs in the nucleus or cytoplasm [12-19]. In the nucleus, lncRNAs can modify gene expression by directly interacting with DNA or chromatin regulators, such as transcription factors and RNA binding proteins, acting as enhancers, decoys, scaffolds or guides. While in the cytoplasm, lncRNAs enable mRNA decay, modulate the stability or translation of mRNAs, compete with microRNA for binding to mRNA and can be processed into microRNAs [20]. Nowadays, lncRNAs are increasingly drawing attention and flourishing evidence has warranted lncRNAs associate with multiple diseases, notably cancer [21]. In cancer cells, IncRNAs are aberrantly expressed as classical oncogenes or tumor suppressors and correlate with the altered metabolism $[1,22]$. Therefore, targeting lncRNAs promises great potential as an alternative and workable therapy for cancer in the context of aberrant metabolism. In this review, we focus on metabolism-related lncRNAs and discuss their regulatory roles in cancer metabolism as well as their potential clinical translation via the regulation of cancer metabolism.

\section{LncRNAs regulate mitochondrial function}

Mitochondria are the center of many biochemical processes including oxidative phosphorylation (OXPHOS), the krebs cycle, intracellular calcium balance, and the synthesis of cytosolic biosynthetic precursors such as amino acids, nucleotides, lipids, heme and NADPH $[23,24]$. As mitochondria play an essential role in multiple cellular biological processes, proper mitochondrial quality control and component integrity is pivotal for cancer maintenance and progression, particularly cancer cell metabolism.

Mitochondria are highly dynamic organelles associated with constant fusion and fission, which affect mitochondria shape, distribution and function $[25,26]$. Mitochondrial dynamics has been found to be involved in cell metabolism [27-30]. For instance, disruption of mitochondrial fusion proteins MFNs or OPA1 causes reversible cellular respiration defects, while downregulation of mitochondrial fission protein DRP1 attenuates mitochondrial respiratory capacity [30, 31]. Moreover, mitochondrial morphology could be adjusted by mitochondrial dynamics change in response to nutrient availability. For example, cancer cells dominant in OXPHOS activity tend to have condensed mitochondria, while those dependent on glycolysis show more orthodox conformation of mitochondria [32]. Thus, exploring the roles lncRNAs play in mitochondria dynamics is of great importance to understand cancer metabolism.

Recently, studies have shown that nuclear-encoded lncRNAs regulate mitochondrial dynamics. FIS1 localizes on mitochondrial outer membrane and acts as a receptor for DRP1 recruitment to promote mitochondrial fragmentation, and FIS1 can induce cytochrome $\mathrm{c}$ dependent apoptosis [33]. Mitochondrial dynamic related lncRNA (MDRL) downregulates miR-361 expression and indirectly upregulates miR-484, a negative regulator of Fis1 protein translation, by reducing the interaction between miR-361 and pri-miR-484. MiR-361 can bind to pri-miR-484 and inhibit its maturation by Drosha into pre-miR-484 in the nucleus, resulting in increased Fis1 and apoptosis. Thus, MDRL indirectly regulates mitochondrial fission and apoptosis [34, 35] (Fig. 1A). In contrast, focally amplified lncRNA on chromosome 1 (FAL1) inhibits apoptosis and cytochrome $\mathrm{c}$ release in esophageal squamous cell carcinoma (ESCC) cells by suppressing DRP1, which promotes cancer cell survival and increases mitochondrial respiration [36]. Fis1 can also be targeted by other microRNAs such as miR-483-5p. In tongue squamous cell carcinoma (TSCC), miRNA processing-related lncRNA (MPRL) can upregulate Fis1 by preventing its upstream regulator miR-483-5p generation from TRBP-DICER-complex mediated recognition and subsequent cleavage of pre-miR-483. As the result, MPRL overexpression increases FIS1 expression to promote mitochondrial fission, inhibit tumor growth and enhance cisplatin sensitivity [37] (Fig. 1B). Though no evidence so far has directly pointed out how lncRNAs regulate cancer cell metabolism by affecting mitochondrial dynamics, it is rational to further investigate into the prospective correlations.

In addition to mitochondrial dynamics, mitochondrial contents related to OXPHOS can also be targeted by lncRNAs in diverse manners. LncRNAs can regulate cell metabolism and cancer cell survival by affecting mitochondria components including complexes I-IV and other subunits such as ATPase on mitochondria inner membrane [38]. Hox Transcript Antisense Intergenic RNA (HOTAIR), one of the few well-studied lncRNAs, is a negative 
prognostic indicator of many cancers such as pancreatic cancer [39]. In Hela cells, HOTAIR knockdown causes mitochondria dysfunction including reduced expression of OXPHOS component Ubiquinol-Cytochrome C Reductase Complex III Subunit VII (UQCRQ) and impairment of OXPHOS activity. Therefore, it results in increased intracellular ROS stress, vacuoles formation, mitochondrial morphology changes shown as swollen with loss of cristae, and mitochondria deprivation [40]. Mitochondrial calcium uptake 1(MICU1) is a core component of mitochondrial calcium uniporter complex for regulating calcium release and maintaining proper mitochondrial membrane potential [41]. HOTAIR blockage in head and neck squamous cell carcinoma (HNSCC) activates MICU1-dependent mitochondria-related apoptosis by reducing Bcl-2 and increasing pro-apoptotic proteins including BAX [42] (Fig. 1C). However, it remains unknown how HOTAIR regulates mitochondria functions. In addition to HOTAIR, SAMMSON, which primarily locates in the cytoplasm and mitochondria, is lincRNA specific for melanoma survival independent of MITF or BRAF, NRAS and p53 status, moreover, it can regulate mitochondria function [43]. SAMMSON silencing causes mitochondria structure aberration and decreases the activity of respiratory complexes I and IV, leading to mitochondrial membrane potential depolarization, mitochondrial precursor-over-accumulation stress (mPOS) and cell apoptosis in melanoma. Mechanistically, the mitochondria part of SAMMSON directly interacts with p32, a protein required for mitochondrial $16 \mathrm{~S}$ rRNA maturation, to enhance its function [44]. As a result, its silencing decreases mitochondrial fraction of p32 to reduce mtDNA-encoded COX2 and ATP6, but not nuclear-encoded SDHA and NDUFS3 or other ETC components, thus inducing fragmented mitochondria cristae and less mitochondria matrix [44] (Fig. 1D). Similarly, lncRNA RMRP can also affect mtDNA-encoded proteins. RMRP is encoded by nuclear DNA and is transported into mitochondria matrix by RNA-binding proteins HuR and GRSF1. RMRP induces mtDNA replication and increases OXPHOS subunit ATP6, COX1 and CYB, which facilitate mitochondrial respiration in Hela cells [45] (Fig. 1E).

Besides being regulated in the cytoplasm, mitochondrial dynamics and function can be modulated from the nucleus. LncRNA nuclear enriched abundant transcript 1 (NEAT1) forms the main structural component of paraspeckles which can mediate the retention of nuclear-encoded mRNA. The middle region of NEAT1_2, one of NEAT1 isoform, is in the core region, surrounded by its $5^{\prime}$ and $3^{\prime}$ ends and another isoform NEAT1_1 [46]. Upon mitochondrial protein depletion or mito-stressor induction, nuclear transcription factor ATF2 binds to the promoter of NEAT1 and enhances the transcription of NEAT1 long-isoform NEAT1_2, promoting the elongation of paraspeckles. Elongated paraspeckles enhance the retention of nuclearencoded mito-mRNAs which encodes proteins involved in mitochondrial function, including cytochrome c, subunit of NADH dehydrogenases and Carnitine O-palmitoyltransferase 1 [47]. Thus, NEAT1 exerts important effect on the regulation of mitochondrial homeostasis. Moreover, NEAT1 depletion leads to mitochondria elongation by inhibiting mitochondrial fission via decreased DRP1 expression and phosphorylation, causing mitochondrial dysfunction including reduction in mtDNA, respiration capacity, ATP output, extracellular acidification rate (ECAR) and reduced cell proliferation [47].

Some transcription factors essential for the transcription of mitochondrial proteins can also be regulated by lncRNAs. Peroxisome proliferator-activated receptor-c coactivator-1a (PGC-1a), one central transcriptional coactivator of genes related to mitochondrial biogenesis and mitochondrial oxidative metabolism, is dysregulated in several cancer types including colorectal cancer [48]. LncRNA taurine-upregulated 1 (Tug1) interacts with tug1-binding element upstream of PGC-1a promotor, triggering PGC-1a transcription and improving mitochondrial bioenergetics. Tug1 knockdown inhibits OXPHOS subunit complex I and III activity, decreases ATP production, increases intracellular ROS and induces apoptosis, which can be rescued by overexpressing PGC-1a [49] (Fig. 1F).

In addition to gene transcription, protein translation can be targeted by lncRNAs to affect cancer metabolism and mitochondrial function. Eukaryotic translation initiation factor 4AIII (eIF4AIII) is an ATPase and RNA helicase that mediates pre-mRNA splicing, translation inhibition and nonsense-mediated decay in the nucleus [50,51]. eIF4AIII has target sites at mRNAs of metabolic enzymes involved in TCA cycle and oxidative phosphorylation, such as PKM, IDH2 and UQCRH. LncRNA SNHG3 is predicted to bind to and inhibit eIF4AIII to favor cancer cell survival. Moreover, SNHG3 sponges miR-186-5 $p$ to inhibit its binding to 3'UTR of PDHB [52] (Fig. 1G).

Collectively, IncRNAs are unprecedented essential regulators of mitochondrial function and metabolism by modifying mitochondrial dynamics and respiration. These regulatory operations exert 
great impact on cellular response to oncogenic stimuli and cancer cell behaviors.

\section{LncRNAs regulate glucose metabolism}

Glucose is the major carbon source for cellular biosynthesis and energy generation, and altered glucose metabolism is one of the first identified hallmarks of cancer [3]. Glucose transporters and multiple enzymes involved in or associated with glucose metabolism in cancers are found to be targeted by lncRNAs through diverse mechanisms (Fig. 2).

To date, many lncRNAs have been reported to be regulated by several well-known transcription factors in response of glucose availability in the environment or serving as mediators to modulate glucose metabolism. Among them, p53 is a frequently mutated or deleted tumor suppressor in various tumors. A number of studies have shown that p53 is closely correlated with cancer metabolism covering glycolysis, oxidative phosphorylation and pentose-phosphate pathway (PPP) [53-55]. p53 can activate the transcription of many lncRNAs in addition to well-known mRNAs. It has been found that p53 directly upregulates lncRNA Tp53-regulated inhibitor of necrosis (TRINGS) in multiple tumor cells after glucose deprivation. TRINGS facilitates tumor growth in vitro and in vivo by binding to STRAP and inhibiting STRAP-GSK3 $\beta-N F-\kappa B$ necrotic pathway [56]. LincRNA-p21, a downstream transcriptional target of p53, can be upregulated upon DNA damage to repress the transcription of p53 targeted genes [57].
(A)

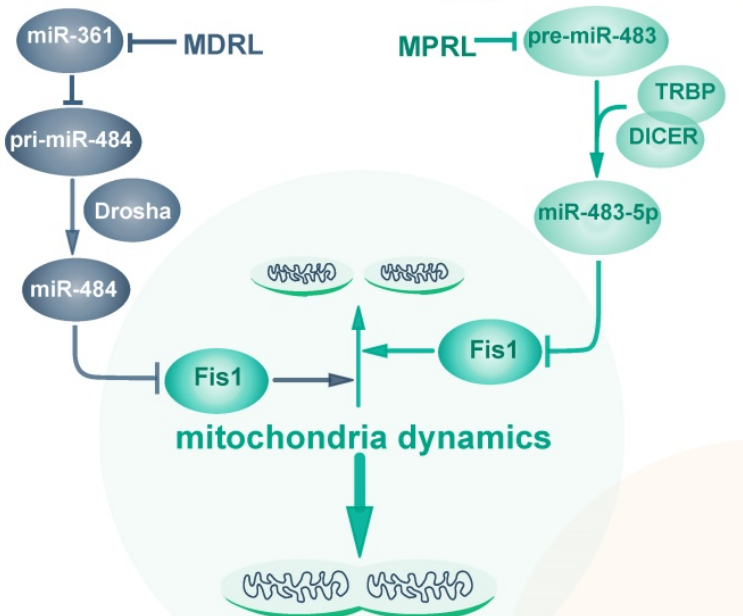

(C)

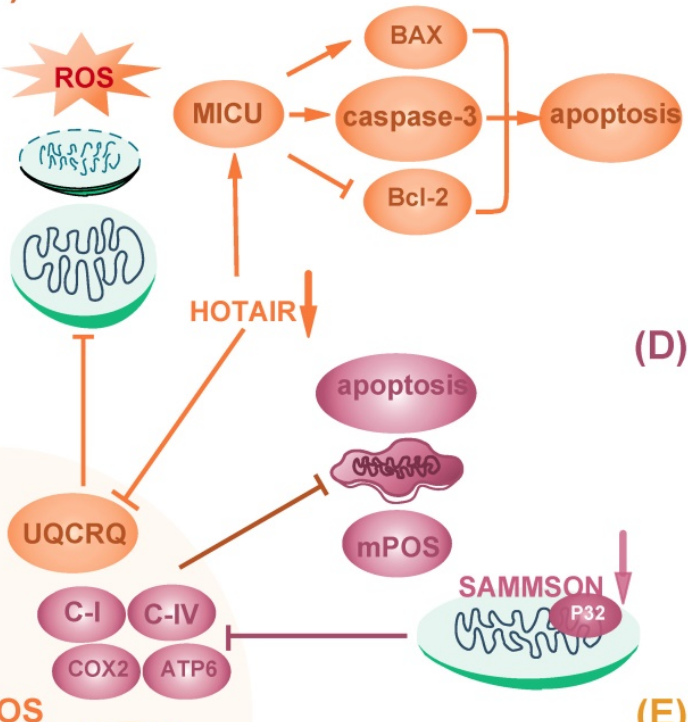

(E)

D)

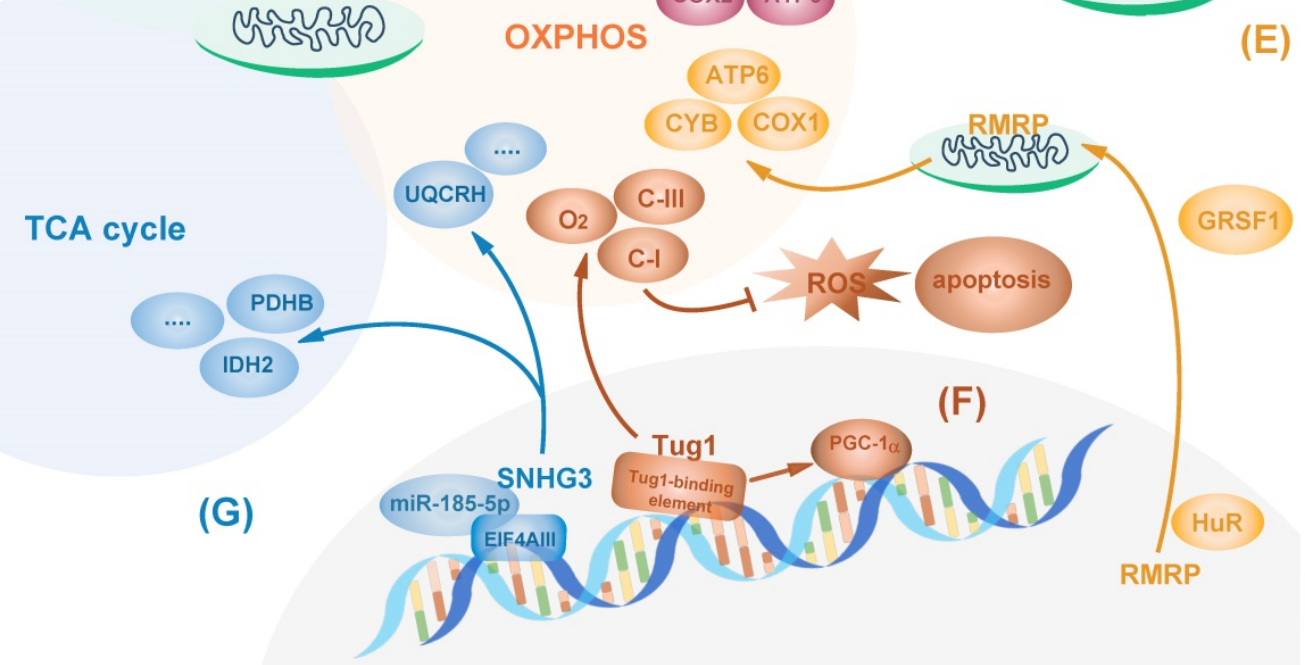

Figure 1. LncRNAs regulate mitochondria function. (A, B) LncRNA MDRL (A) and MPRL (B) act as sponges of miR-484 and miR-483-5p, respectively, which targets Fis 1 to inhibit its expression and regulate mitochondrial dynamics. (C) HOTAIR knockdown impairs mitochondrial function via reducing its OXPHOS components including UQCRQ and triggers ROS stress. Also, HOTAIR blockage activates MICU and induces apoptosis by reducing Bcl-2 and increasing caspase 3 and BAX. (D) Mitochondrial part of SAMMSON inhibits mitochondrial membrane potential depolarization, mPOS and tumor apoptosis by interacting with p32 and enhancing its function. (E) Nuclear encoded RMRP transports into mitochondria facilitated by HuR and GRSF1 to induce mtDNA replication and increase OXPHOS subunit, thus promoting mitochondrial respiration. (F) Tug 1 interacts with tugl-binding element upstream of PGC-1a promoter to maintain complex I and III activity and increase mitochondrial bioenergetics. (G) SNHG3 sponges miRNA-186-5p and interacts with EIF4AIII to regulate gene expression related to TCA cycle and OXPHOS activity such as PDHB, IDH2 and UQCRH. 
LincRNA-p21 knockdown upregulates PKM2 expression through PTEN/AKT/mTOR pathway, thus activating glycolysis and enabling prostate cancer progression [58]. Moreover, lincRNA-p21 is upregulated by HIF-1a and reciprocally binds to HIF-1a, preventing HIF-1a from degradation by interrupting HIF-1a-VHL interaction. Therefore, it can promote HIF-1a accumulation and hypoxia-enhanced glycolysis [59].

C-Myc is another oncogenic transcription factor upregulated in a wide variety of cancers. It directly or indirectly targets genes involved in glycolysis, including LDHA, GLUT1, HK2, PFKM, and ENO [60]. LncRNA prostate cancer gene expression marker 1 (PCGEM1) is specifically expressed in prostate cancer and can be induced by androgen. PCGEM1 acts as a coactivator of c-Myc and facilitates c-Myc as well as other regulators to transcriptionally activate PCGEM1-targeted metabolic genes by increasing histone $\mathrm{H} 3$ and $\mathrm{H} 4$ acetylation. Besides, PCGEM1 knockdown downregulates various metabolic genes and inhibits glycolysis via unknown transcription factors, revealing another layer of metabolic regulation independent of androgen signaling [61]. In colon cancer, linc00504 could promote cell progression both in vivo and in vitro by regulating multiple metabolic pathways, including glucose metabolism, at transcriptional level. It interacts with c-Myc to promote its chromatin recruitment and enhance its transactivation activity [62]. Recently, FoxO-induced long non-coding RNA 1 (FILNC1) has been reported to repress the transcription of glycolysis genes, including glucose transporter 1 and 3 (GLUT1/3), hexokinase 2 (HK2), aldolase C (ALDOC), lactate transporter MCT4 as well as pyruvate dehydrogenase kinase 1 (PDK1) and PDK4. In detail, FILNC1 suppresses the translation of c-Myc mRNA by sequestering AUF1, which is further enhanced under glucose starvation. Thus, FILNC1 downregulation enhances Warburg effect and indicates poor prognosis in renal cell carcinoma [63].

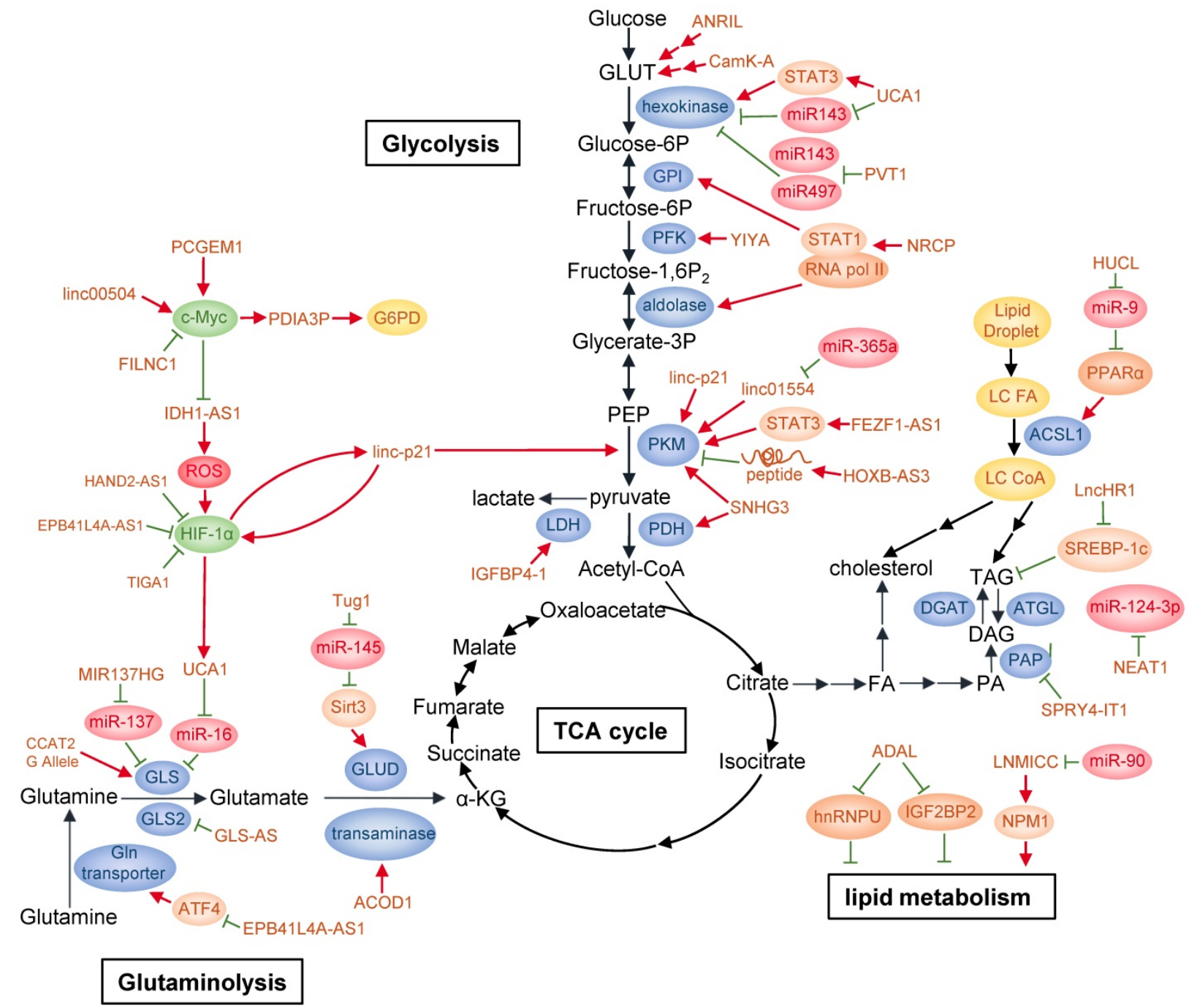

Figure 2. LncRNAs regulate cancer metabolism. By regulating glycolysis, glutaminolysis, and lipid metabolism, IncRNAs can reprogram major cancer metabolism pathways. Detailed mechanisms of action of these IncRNAs are described in the text. 
In addition, lncRNAs can activate the transcription of metabolic enzymes directly or indirectly. For example, in bladder cancer, lncRNA urothelial carcinoma associated 1 (UCA1) is transcriptionally upregulated by HIF-1a under hypoxia [64]. It can stimulate glycolysis indirectly through UCA1-mTOR-STAT3/miR143-HK2 cascades, in which STAT3 transcriptionally upregulates HK2 mRNA level and miR143 reduction increases hexokinase 2 (HK2) protein level [65]. Antisense non-coding RNA at the INK4 locus (ANRIL) promotes AML progression by regulating adiponectin receptor1 (AdipoR1)/AMPKa/SIRT1 pathway, elevating GLUT1 and LDHA expression to promote AML cell survival [66]. Under glucose deprivation, lncRNA insulin-like growth factor binding protein 4-1 (IGFBP4-1) induces ATP production and increases expression of HK2, PDK1 and LDHA at transcriptional level to promote glucose metabolism and tumor progression in lung cancer, though the detailed molecular mechanism was not investigated [67]. Besides its role in OXPHOS mentioned above, SNHG3 also regulates glycolysis and TCA cycle in ovarian cancer at transcriptional level by targeting genes such as pyruvate kinase $\mathrm{M}$ (PKM), pyruvate dehydrogenase E1 component subunit beta (PDHB) and isocitrate dehydrogenase 2 (IDH2) [52]. Moreover, ceruloplasmin (NRCP), a lncRNA significantly overexpressed in ovarian cancer, increases the interaction between RNA polymerase II and STAT1 in the nucleus, which enhances the transcription of enzymes involved in glycolysis such as glucose-6-phosphate isomerase (GPI), ALDOA and ALDOC [68]. In a siRNA library screening, lncRNA for calcium-dependent kinase activity (CamK-A) was found to affect glycolysis and cell survival in various cancer types. In response to hypoxia, increased cytosolic calcium flux from endoplasm reticulum induces CamK-A upregulation. CamK-A activates pregnancy upregulated non-ubiquitously expressed CaM kinase (PNCK) and phosphorylates IKBa, subsequently activating the transcription of genes downstream of NF-kB, including GLUT3 for enhanced glucose uptake and cytokines for tumor microenvironment remodeling such as microphage infiltration and angiogenesis [69]. LncRNA metastasis-associated lung adenocarcinoma transcript 1 (MALAT1) modulates both gluconeogenic and glycolytic genes including GLUT1, HK2, ENO1 and PKM2 transcriptionally by translationally increasing transcription factor TCF7L2 protein level in a mTORC1-4EBP1 axis mediated cap-dependent manner [70].

Accumulating evidence has indicated that lncRNAs can act as competing endogenous RNAs
(ceRNAs) to regulate glucose metabolism. LncRNA H19 was previously reported to be oncogenic in glioma, hepatocellular carcinoma (HCC) and bladder cancer, but none of them were related to cancer metabolism [71-74]. A recent study found that lncRNA H19 functions as a ceRNA of miR-106a-5p and competitively binds to E2F3, a transcription factor facilitating the expression of glucose metabolic genes, thus promoting glucose metabolism in melanoma and serving as an indicator of poor prognosis [75]. Upon glucose starvation, lncRNA Heart and Neural Crest Derivatives Expressed 2-antisense 1 (HAND2-AS1) downregulation significantly induces glycolysis, decreases apoptosis as well as increases cell viability and colony formation in osteosarcoma. Acting as a ceRNA, HAND2-AS1 facilitates fructose-1, 6-bisphosphatase 1 (FBP1) bind to HIF-1a mRNA, which decreases HIF-1a mRNA level and inhibits the transcription of metabolic genes such as GLUT1, GLUT3, HK2, ALDOC and MCT4 downstream of HIF-1a [76]. In osteosarcoma, lncRNA PVT1 induces glycolysis via inhibiting the negative regulation of HK2 mRNA by miR-497 [77]. In gallbladder cancer (GBC), PVT1 positively regulates HK2 expression by sponging miR-143 [78]. Therefore, PVT1 regulates glycolysis through competing with different microRNAs in a cancer type-specific manner.

LncRNAs also regulate glycolytic enzyme function by affecting protein modification. YIYA or linc00538 increases 6-phosphofructo-2-kinase/ fructose-2, 6-biphosphatase 3 (PFKFB3) enzymatic activity through CDK6-dependent phosphorylation, thereby enhancing the conversion of G6P/F6P to FBP/GBP. Increased expression of YIYA and CDK6 maintain elevated glycolysis in breast cancer, thus disfavoring patients' survival [79]. LncRNA IDH1 antisense RNA1 (IDH1-AS1) enhances IDH1 enzymatic activity at post-translational level by facilitating IDH1 homodimerization, resulting in increased production of a-ketoglutarate (a-KG) as well as downregulation of ROS [80, 81]. Under hypoxia, PHDs catalyzes HIF1a hydroxylation, a prerequisite for its conjugation to von Hippel-Lindau (VHL) and subsequent degradation through ubiquitin-proteasome pathway [82]. However, the hydroxylaton of HIF-1a could also be regulated under normoxia by variant stimulants, such as TCA cycle intermediates a-ketoglutarate, succinate, fumarate and malate [81]. Thus, IDH1-AS1 can indirectly activate HIF-1a by increased a-KG under normoxia. IDH1-AS1 is also transcriptionally repressed by c-Myc [80]. This c-Myc- (IDH1-AS1)-IDH1-aKG/ROS-HIF1a axis links two of the most important cancer metabolism effectors together, indicating the complexity of metabolic regulation network. 
LncRNAs can also affect glycolytic enzyme degradation. LINC01554, negatively regulated by miR-365a in HCC and correlated with poor clinical outcomes, suppresses aerobic glycolysis by decreasing PKM2 level through ubiquitin-proteasome pathway and inhibiting Akt/mTOR pathway in the cytoplasm [83]. FEZF1-AS1 promotes cancer maintenance and metastasis in colorectal cancer by binding to and increasing PKM2 abundance through transcription in the nucleus, or protecting PKM2 from degradation in proteasome pathway in the cytoplasm. In the nucleus, upregulated PKM2 activates STAT3 pathway, which further enhances PKM transcription, while accumulated cytoplasmic PKM2 enables increased PKM2 tetramer formation, which promotes pyruvate production from phosphoenolpyruvate (PEP) [84].

As indicated by the name, most lncRNAs do not encode peptides [85]. However, this concept was overturned in recent studies. For example, lncRNA HOXB cluster antisense RNA 3 (HOXB-AS3) encodes a 53-aa peptide, which suppresses colorectal cancer (CRC) progression. Mechanistically, the HOXB-AS3 peptide reduces PKM2 transcription by selectively binding to hnRNP A1 binding domain directed at the sequences flanking PKM exon 9. Thus, lactate production is reduced and glucose metabolism is suppressed. Consistently, colon cancer tissues of various stages conformably show decreased mRNA and protein levels of HOXB-AS3 [86].

Pentose phosphate pathway, bifurcated from glycolysis, is an important source of intracellular NADPH and ribonucleotides through oxidation, and pentose phosphates by non-oxidation, which cope with oxidative stress and supply materials for nucleic acid and fatty acid synthesis [87]. PPP has been reported to be dysregulated in cancers to favor proliferation and metastasis, and is regulated by multiple oncogenes such as TP53 and mTORC1 [88, 89]. Glucose-6-phosphate dehydrogenase (G6PD) is a rate-limiting enzyme that catalyzes dehydrogenation of glucose-6-phosphate (G6P) into NADPH and 6-phosphogluconolactone. G6PD expression is transcriptionally activated by c-Myc, which is further enhanced by IncRNA protein disulfide isomerase family A member 3 pseudogene 1 (PDIA3P) via interacting with c-Myc. Thus, PDIA3P promotes PPP and enables multiple myeloma progression [90]. Another study showed that IncRNA prostate cancer gene expression marker 1 (PCGEM1) binds to target promoters through interacting with c-Myc and increasing its chromatin recruitment, thus enhances c-Myc transactivation activity and affects multiple metabolism pathways including PPP [61].
Targeting enhanced glucose metabolism in cancer has already been clinically investigated to develop therapeutic drugs or available diagnostic tools. The various aspects of lncRNAs involved in glucose metabolism denote a vital way to further the insight into cancer progression. Therefore, lncRNAs possess great potential in developing effective therapies and diagnostic approaches against dysregulated glucose metabolism in cancer.

\section{LncRNAs regulate glutamine metabolism}

Pyruvate influx into mitochondria is the initial step to elicit TCA cycle. In cancer cells, the transportation of pyruvate into mitochondria is drastically decreased due to increased lactate production. To maintain mitochondrial function and essential TCA cycle that supply macromolecules required for cancer cell proliferation, metabolic intermediates flowing into the mitochondria need replenishment from alternative sources. Glutamine, the most abundant amino acid in human body, is involved in most biosynthetic and bioenergetic pathways in many cancer cells. In this way, glutamine serves as an important carbon or nitrogen source through mitochondrial anaplerosis [91]. Once transported into the cytoplasm, glutamine is catalyzed into glutamate by glutaminase (GLS1/GLS2), then converted into a-KG, an intermediate in TCA cycle, via glutamate dehydrogenase (GLUD/GDH) or transaminases, coupled with production of NADH, NADPH, ammonium, and other nonessential amino acids (NEAAs). Thus, glutamine provides precursors, such as citrate and oxaloacetate, for the synthesis of amino acids, nucleotides and fatty acids. Glutamine also donates amide ( $\gamma$-nitrogen) group for NEAAs, purine and pyrimidine synthesis [91, 92]. Besides, glutamine is an important source of glutathione, a dominant factor for relieving intracellular redox stress. Therefore, glutamine plays a widespread role in cellular activity, and lncRNAs also aim at several enzymes in glutamine metabolism (Fig. 2).

Glutaminase is the rate-limiting enzyme in glutaminolysis and is subjected to fine-tuned regulation. It is generated mainly from GLS1 and the two isoforms glutaminase isoform $C$ (GAC) and glutaminase kidney isoform (KGA) differ in their catalytic ability. LncRNA Colon Cancer-Associated Transcript 2 (CCAT2) interacts with cleavage Factor I (CFIm) subunits in an allele-specific manner. The G allele of CCAT2 at rs6983267 SNP site preferentially binds to CFIm25 rather than CFIm68. The former complex interacts with GLS pre-mRNA and leads to increased splicing of GLS mRNA into GAC, resulting in increased glutamine metabolism. Cancer cell with 
CCAT2 G allele display more activated cellular metabolism of glucose, TCA cycle, glutamine and fatty acids. As a result, rs6983267 SNP with G allele, rather than $\mathrm{T}$ allele, is associated with greater risk in colorectal cancer [93]. Heat Shock Factor 1 (HSF1) can recruit DNMT3a to methylate the promoter of lncRNA MIR137HG and increase its transcription, which subsequently represses the expression of miR-137. GLS1 mRNA can be targeted and suppressed by miR-137. As a result, HSF1 stimulates GLS1-dependent glutaminolysis and activates mTOR to promote colorectal carcinogenesis [94]. Besides GLS1, GLS2 is also related to reprogrammed glutaminase activity in cancer. LncRNA urothelial carcinoma-associated 1 (UCA1) interferes the negative regulation of GLS2 mRNA by miR-16, upregulating GLS2 expression to promote glutaminolysis, inhibit ROS production and protect cells from oxidative toxicity in bladder cancer [95-97].

GLS-AS is a nucleus-located antisense lncRNA of GLS. In pancreatic cancer cells, upon glucose and glutamine deprivation, GLS-AS is downregulated by c-Myc at transcriptional level and reduces its binding to GLS pre-mRNA, which inhibits GLS pre-mRNA degradation via ADAR/Dicer-dependent RNA interference. Thus, GLS is upregulated at post-transcriptional level and promotes pancreatic cancer cell survival as well as metastasis. Conversely, c-Myc protein stability is impaired partially in proteasome pathway by GLS-AS in a GLS-dependent manner, hence forming a negative feedback loop between c-Myc and GLS-AS [98].

Several studies have shown that transaminases are regulated by lncRNAs. A special viral infection-induced lncRNA aconitate decarboxylase 1 (ACOD1) directly interacts with glutamic-oxaloacetic transaminase-2 (GOT2) to enhance its enzymatic activity and provide metabolites for viral replication [99]. ACOD1 expands lncRNA function in response to various stimulants, indicating that lncRNAs are involved in a wide regulatory network. Given that several types of cancer including HCC are associated with viral infection, the regulatory role of lncRNA found in viral infection may provide a new therapeutic target for such cancers. Another study showed that lncRNA taurine upregulated gene 1 (Tug1) prevents miR-145 mediated degradation of Sirt3 mRNA, promoting Sirt3 protein expression. Sirt3 positively regulates GDH translation and enhances glutamine consumption, a-KG and ATP production in intrahepatic cholangiocarcinoma (ICC) [100]. Thus, Tug1 indirectly upregulates GDH to assist glutamine metabolism.

Studies also reveal that lncRNAs can regulate the transcription of intermediates in glutamine metabolism or other energetic metabolism through epigenetic modification. LncRNA EPB41L4A-AS1 is involved in both glycolysis and glutaminolysis, and its low expression correlates with poor clinical prognosis. EPB41L4A-AS1 predominantly enhances the interaction between HDAC2 and NPM1 in nucleolus, which prevents HDAC2 nucleoplasm translocation and subsequent occupation on VHL and VDAC1 promoters. Therefore, VHL and VDAC1 transcription was increased via histone acetylation, resulting in reduced HIF-1a protein levels and inactivated downstream p-eIF2a/ATF4 pathway, respectively. As HIF-1a could transcriptionally upregulate glycolytic genes and ATF4 activates the expression of amino acid transporters, EPB41L4A-AS1 eventually inhibits glycolysis and glutaminolysis in cancer cells. Moreover, TIGA1, a small protein generated by EPB41L4A-AS1, locates on mitochondrial outer membrane and mediates the connection between a-tubulin and mitochondria. Knockdown of TIGA1 promotes microtubule depolymerization via reduced acetylated a-tubulin, which induces cellular stress and ROS production due to VDAC block by binding with free tubulin. Consequently, p38 is phosphorylated and subsequently increases HIF-1a level [101].

To sum up, lncRNAs function to regulate glutamine metabolism at transcriptional or post-transcriptional level through different mechanisms, and the various properties of lncRNAs contribute to the complexity and multifaceted regulation of glutamine metabolism.

\section{LncRNAs regulate lipid metabolism}

Reprogrammed lipid metabolism in various tumors plays an oncogenic role in multiple aspects of tumorigenesis, including metastasis, interaction with tumor microenvironment, redox stress balance, drug resistance, energy supply, and homeostasis maintenance [102-104]. Lipids provide carbon derived from glucose and glutamine for basic cellular framework construction. Moreover, lipids produce energy in nutrient-deficient microenvironment and help modulate redox status by NADPH, one of the consumables in lipid synthesis pathway [104] (Fig. 2).

Sterol regulatory element binding proteins (SREBPs) are key transcription factors that regulate genes for lipid synthesis or lipid transportation in response to fluctuant intracellular lipid level [105]. Induced upon HCV infection, lncHR1 suppresses SREBP-1c expression and subsequent fatty acid synthase (FAS) activity, thereby decreasing the abundance of oleic acid-induced triglyceride (TG) and lipid droplet (LD) in hepatoma cells [106]. Peroxisome proliferator-activated receptor a (PPARa) is another 
transcription factor that regulates genes involved in lipid metabolism and its expression is implicated in inflammation, fatty acid oxidation, tumorigenesis, and drug resistance [107]. LncRNA nuclear paraspeckle assembly transcript 1 (NEAT1) interferes HCC lipolysis and promotes HCC growth in vitro and in vivo by transcriptionally maintaining high levels of adipose triglyceride lipase (ATGL) and its catalytic products, diacylglycerol (DAG) and free fatty acid (FFA). In addition, NEAT1 increases fatty acid oxidation (FAO) via the activation of PPARa signaling pathway directly by ATGL or indirectly by DAG and FFA, which is attenuated by transcriptional inhibition of ATGL by miR-124-3p, a downstream effector of NEAT1. Moreover, TP53 positively targets NEAT1 and in turn TP53 expression is suppressed by NEAT1 in HCC [108]. However, in other cancer types, NEAT1 is highly expressed independent of p53 or is downregulated by p53 [109, 110].

In addition to the regulation of pivotal lipid transcription factors, lncRNAs also regulate lipid metabolism through other mechanisms. For example, linc- adipogenesis and lipogenesis (ADAL) interacts with hnRNPU in the nucleus and IGF2BP2 in the cytoplasm, affects a subset of lipogenic gene expression at transcriptional or post-transcriptional level and regulates pre-adipocyte differentiation as well as de novo fatty-acid biosynthesis in mature adipocytes, thereby altering lipid storage and fatty acid oxidation [111]. Acetyl-CoA synthase long-chain 1 (ACSL1) promotes fatty acids uptake and catalyzes the first step in long-chain FA synthesis pathway. One study showed lncRNA HUCL depresses miR-9 targeted PPARa inhibition, then activates expression of ACSL1 and leads to accumulation of cholesterol and triglycerides in hepatoma [112]. Another study showed that lncRNA LNMICC, which is negatively regulated by miR-190, recruits transcription factor NPM1 to the promoter of fatty acid binding protein 5 (FABP5) in the nucleus to enhance fatty-acid metabolism. Furthermore, LNMICC correlates with lymph node metastasis, epithelial-mesenchymal transition and lymphangiogenesis in cervical cancer [113].

\section{LncRNAs regulate tumor microenvironment}

Besides synthesized intracellularly, lncRNAs are also exported out of cancer cells or cancer-associated fibroblasts, capsuled in the exosomes and then incorporated by adjacent cells, thus setting up an intercellular signaling pathway and altering tumor microenvironment to modulate tumor metabolism $[114,115]$.
In addition to function in an autocrine manner, lncRNAs can be regulated by stromal cells in tumor environment. Cancer-associated fibroblasts (CAFs) are a group of tumor stromal mensenchymal cells exhibiting common hallmarks but possessing heterogeneous properties and functions. Moreover, CAFs correlate with tumor initiation, progression and metastasis by modulating tumor microenvironment, and providing nutrients and chemokines via paracrine signaling pathway [116]. For example, CXCL14, a pro-metastatic chemokine secreted by CAFs, promotes ovarian cell invasion by upregulating a long intergenic RNA LINC00092 in ovarian cancer cells. LINC00092 enhances glycolysis in ovarian cancer by binding to glycolytic enzyme fructose-2, 6-biphosphatase (PFKFB2) and activating 6-phosphofructo-1-kinase (PFK-1) via PFKFB2 catalytic product fructose-2, 6-bisphosphate (F-2, 6-BP). In turn, CXCL14 maintains the CAFs-like phenotype, thus forming a positive feedback circuit and playing a critical role in cancer metastasis [117] (Fig. 3A). In addition, CXCL14 is also excreted by glioblastoma-associated stromal cells (GASCs) and elevates lncRNA UCA1 expression in cancer cells to activate PFKFB2 by sequestering miR-182 from binding to PFKFB2 mRNA [118] (Fig. 3B). Conversely, lncRNAs can contribute to the aberrant reprogramming in CAFs. One study showed that IncRNA-CAF (Lnc-CAF), synthesized in stromal fibroblasts or internalized from tumor cell-excreted exosomes, specifically mediates the transformation of normal fibroblasts into CAFs in oral squamous cell carcinoma (OSCC) by upregulating IL-33, a chemokine previously reported to reduce apoptosis, promote metastasis, and enable escape from immune system in tumors $[119,120]$. Lnc-CAF prevents IL-33 degradation through p62-mediated autophagylysosome pathway, thus inhibits CAFs autophagy and induces angiogenesis. As a result, lncRNA-CAF supports tumor growth in a positive feedback manner [119] (Fig. 3C). However, whether lnc-CAF induced fibroblast transition correlates with metabolic reprogramming awaits further investigation.

Besides, lncRNAs can induce metabolic reprogramming by mediating interaction between tumor cells and tumor-associated macrophages (TAMs). In breast cancer, upon lactate stimulation, TAMs synthesize and secret HIF-1a-stabilizing long noncoding RNA (HISLA) wrapped in extracellular vesicles. HISLA can be internalized by breast cancer cells and abolishes HIF-1a degradation by competitively binding to PHD2 and interfering HIF-1a hydroxylation, thereby promoting aerobic glycolysis and increasing chemoresistance of tumor 


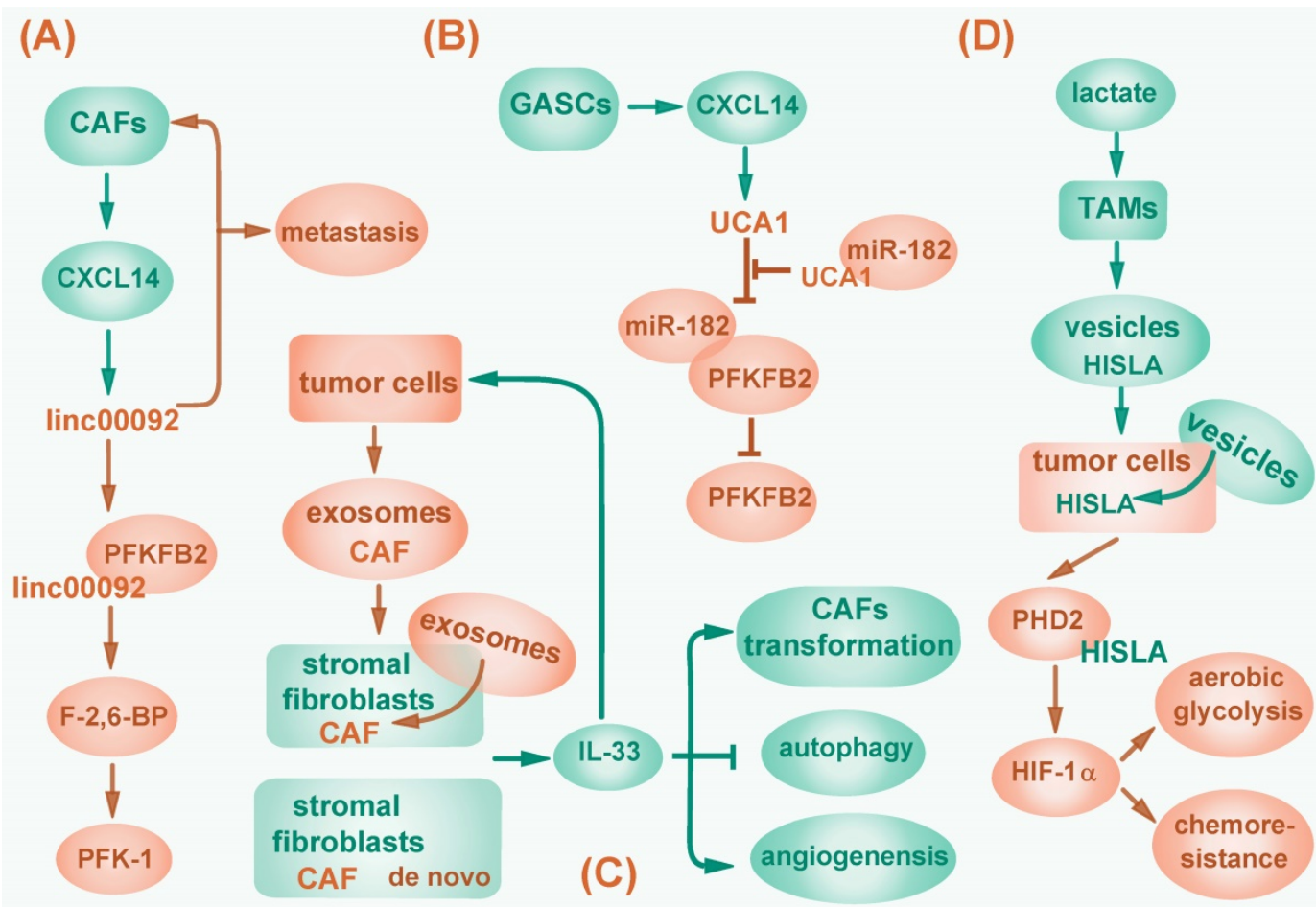

Figure 3. Regulation of cancer-associated fibroblasts by IncRNAs. (A) CXCL14 secreted by CAFs upregulates LINC00092, which binds to PFKFB2 to activate PFK-1 via PFKFB2 catalytic product F-2, 6-BP and then promotes glycolysis. LINC00092 also maintains CAFs-like phenotype and mediates cancer metastasis. (B) CXCL14 secreted by GASCs elevates IncRNA UCA1 expression, then sponges miR-182 to activate PFKFB2. (C) LncRNA-CAF is synthesized in stromal fibroblasts or imported from tumor cell-excreted exosomes to upregulate IL-33, which promotes tumor cell metastasis, mediates CAFs transformation, inhibits CAFs autophagy and induces angiogenesis. (D) Upon lactate stimulation, TAMs synthesize and secret IncRNA HISLA wrapped in vesicles, which are then internalized by cancer cells and abolish HIF-1 $\alpha$ degradation by competitively binding to PHD2, consequently promotes glycolysis and chemoresistance.

cells, which correlates with poor patient prognosis [115] (Fig. 3D).

Taken together, both intracellularly and extracellularly derived-lncRNAs regulate metabolism, consequently, they impact the behavior of cancer cells and modulate tumor microenvironment.

\section{Perspectives and challenges of lncRNAs in cancer metabolism}

So far, lncRNAs have been studied as candidates of cancer molecular biomarkers, contributors to drug resistance and disease progression, as well as potential drug targets along the metabolic pathway. For instance, locked nucleic acid (LNA)-modified antisense oligonucleotides (GapmeRs) could specifically silence SAMMSON and abolish drug resistance to BRAF (V600E) inhibitor dabrafenib and MEK inhibitor pimasertib [121]. Liposomal carrier DOPC nanoparticle with incorporated siRNA was also applied to alleviate NRCP-promoted glycolysis in ovarian cancer [68]. Different from protein-coding genes, which are mainly monitored based on protein expression in the circulating systems or in the pathological tissues, IncRNAs are detected by PCR with high sensitivity, implying their distinct and possibly more broad applications, particularly in differentiating rare diseases at early stages. However, the development of lncRNA-based clinical applications encounters several obstacles and challenges. For example, although quantities of researches are focusing on the function of lncRNAs in cancer, the intricate mechanisms involved in cancer metabolism require further investigations as only a small fraction of lncRNAs are well elucidated and documented. Moreover, inhibition of oncogenic lncRNAs by siRNA-based approach needs efficient delivering system such as biomolecular material packed nanoparticles. Further optimizations are needed to enable these nanoparticles act precisely on tumor cells. Lastly, some lncRNAs regulate cellular metabolism in non-cancer diseases, such as Tug1 in diabetic nephropathy [49] or Pvt1 in skeletal muscle [122]. It will be interesting to study these lncRNAs in tumor models. It is worth noting that the progression in developing diverse analytical methods would enable efficient screening of differentially expressed lncRNAs in cancer and help excavating the underlying mechanisms in cancer metabolism, which hopefully could offer access to clinical applications.

Dysregulated cancer metabolism, a long-discovered phenomenon, is crucial in altered behavior of cancer cells. LncRNAs exert an extensive and complex influence on cancer metabolic effectors and pathways either directly or indirectly, which 
expands our understanding towards the scope of cancer metabolic reprogramming. Therefore, digging into the functions and mechanisms of IncRNAs in cancer metabolism will be helpful for the identification of new biomarkers and therapeutic targets for cancer treatment.

\section{Acknowledgements}

This work is supported in part by the Chinese NSFC grant 31701167 and 81903061, Natural Science Foundation of Zhejiang Province grant LY17C070001, Zhejiang Medical Technology and Education grants 2017KY417 and 2018KY476, Fundamental Research Funds for the Central Universities 2018FZA7005.

\section{Competing Interests}

The authors have declared that no competing interest exists.

\section{References}

1. Hanahan D, Weinberg RA. Hallmarks of cancer: the next generation. Cell. 2011; 144: 646-74.

2. Xiao ZD, Zhuang L, Gan B. Long non-coding RNAs in cancer metabolism. BioEssays : news and reviews in molecular, cellular and developmental biology. 2016; 38: 991-6.

3. Warburg O. Origin of Cancer Cells. Science. 1956; 123: 309-14.

4. Zhou Q, Wang W, He X, Zhu X, Shen Y, Yu Z, et al. Mechanistic study on the nuclear modifier gene MSS1 mutation suppressing neomycin sensitivity of the mitochondrial 15S rRNA C1477G mutation in Saccharomyces cerevisiae. PloS one. 2014; 9: e90336.

5. Vander Heiden MG. Targeting cancer metabolism: a therapeutic window opens. Nature reviews Drug discovery. 2011; 10: 671-84.

6. Zhou Q, Li H, Li Y, Tan M, Fan S, Cao C, et al. Inhibiting neddylation modification alters mitochondrial morphology and reprograms energy metabolism in cancer cells. JCI insight. 2019; 4.

7. Zhu J, Thompson CB. Metabolic regulation of cell growth and proliferation. Nature reviews Molecular cell biology. 2019.

8. Zhou Q, Sun Y. MLN4924: additional activities beyond neddylation inhibition. Molecular \& cellular oncology. 2019; 6: e1618174.

9. Djebali S, Davis CA, Merkel A, Dobin A, Lassmann T, Mortazavi A, et al. Landscape of transcription in human cells. Nature. 2012; 489: 101-8.

10. Iyer MK, Niknafs YS, Malik R, Singhal U, Sahu A, Hosono Y, et al. The landscape of long noncoding RNAs in the human transcriptome. Nature genetics. 2015; 47: 199-208.

11. Ponting CP, Oliver PL, Reik W. Evolution and functions of long noncoding RNAs. Cell. 2009; 136: 629-41.

12. Sun M, Kraus WL. From discovery to function: the expanding roles of long noncoding RNAs in physiology and disease. Endocr Rev. 2015; 36: 25-64.

13. Rinn JL, Chang HY. Genome Regulation by Long Noncoding RNAs. In: Kornberg RD, editor. Annual Review of Biochemistry, Vol 81; 2012. p. 145-66.

14. Fatica A, Bozzoni I. Long non-coding RNAs: new players in cell differentiation and development. Nat Rev Genet. 2014; 15: 7-21.

15. Thomson DW, Dinger ME. Endogenous microRNA sponges: evidence and controversy. Nat Rev Genet. 2016; 17: 272-83.

16. Struhl K. Transcriptional noise and the fidelity of initiation by RNA polymerase II. Nat Struct Mol Biol. 2007; 14: 103-5.

17. Liu G, Zheng $\mathrm{X}, \mathrm{Xu} \mathrm{Y,} \mathrm{Lu} \mathrm{J,} \mathrm{Chen} \mathrm{J,} \mathrm{Huang} \mathrm{X.} \mathrm{Long} \mathrm{non-coding} \mathrm{RNAs}$ expression profile in HepG2 cells reveals the potential role of long non-coding RNAs in the cholesterol metabolism. Chin Med J (Engl). 2015; 128: 91-7.

18. Rapicavoli NA, Ou K, Zhang J, Mikhail M, Laberge RM, Chang HY. A mammalian pseudogene IncRNA at the interface of inflammation and anti-inflammatory therapeutics. eLife. 2013; 2: e00762.

19. Du Z, Fei T, Verhaak RG, Su Z, Zhang Y, Brown M, et al. Integrative genomic analyses reveal clinically relevant long noncoding RNAs in human cancer. Nat Struct Mol Biol. 2013; 20: 908-13.

20. Zhang K, Shi ZM, Chang YN, Hu ZM, Qi HX, Hong W. The ways of action of long non-coding RNAs in cytoplasm and nucleus. Gene. 2014; 547: 1-9.

21. Esposito R, Bosch N, Lanzos A, Polidori T, Pulido-Quetglas C, Johnson R. Hacking the Cancer Genome: Profiling Therapeutically Actionable Long Non-coding RNAs Using CRISPR-Cas9 Screening. Cancer cell. 2019; 35: 545-57.

22. Liu H, Luo J, Luan S, He C, Li Z. Long non-coding RNAs involved in cancer metabolic reprogramming. Cellular and molecular life sciences : CMLS. 2019; 76: $495-504$.
23. Wallace DC. Mitochondria and cancer. Nature reviews Cancer. 2012; 12 . 685-98.

24. Mishra P, Chan DC. Metabolic regulation of mitochondrial dynamics. The Journal of cell biology. 2016; 212: 379-87.

25. Detmer SA, Chan DC. Functions and dysfunctions of mitochondrial dynamics. Nature reviews Molecular cell biology. 2007; 8: 870-9.

26. Westermann B. Mitochondrial fusion and fission in cell life and death. Nature reviews Molecular cell biology. 2010; 11: 872-84.

27. Renault TT, Floros KV, Elkholi R, Corrigan KA, Kushnareva Y, Wieder SY, et al. Mitochondrial shape governs BAX-induced membrane permeabilization and apoptosis. Molecular cell. 2015; 57: 69-82.

28. Montessuit S, Somasekharan SP, Terrones O, Lucken-Ardjomande S, Herzig S, Schwarzenbacher $\mathrm{R}$, et al. Membrane remodeling induced by the dynamin-related protein Drp1 stimulates Bax oligomerization. Cell. 2010; 142: 889-901.

29. Taguchi N, Ishihara N, Jofuku A, Oka T, Mihara K. Mitotic phosphorylation of dynamin-related GTPase Drp1 participates in mitochondrial fission. J Biol Chem. 2007; 282: 11521-9.

30. Chen H, Chomyn A, Chan DC. Disruption of fusion results in mitochondrial heterogeneity and dysfunction. J Biol Chem. 2005; 280: 26185-92.

31. Parone PA, Cruz SD, Tondera D, et al. Preventing mitochondrial fission impairs mitochondrial function and leads to loss of mitochondrial DNA. PLoS One. 2008; 3: e3257.

32. Rossignol R, Gilkerson R, Aggeler R, Yamagata K, Remington SJ, Capaldi RA. Energy substrate modulates mitochondrial structure and oxidative capacity in cancer cells. Cancer research. 2004; 64: 985-93.

33. James DI, Parone PA, Mattenberger Y, Martinou JC. hFis1, a novel component of the mammalian mitochondrial fission machinery. The Journal of biological chemistry. 2003; 278: 36373-9.

34. Wang K, Sun T, Li N, Wang Y, Wang JX, Zhou LY, et al. MDRL IncRNA regulates the processing of miR-484 primary transcript by targeting miR-361. PLoS Genet. 2014; 10: e1004467.

35. Wang K, Long B, Jiao JQ, Wang JX, Liu JP, Li Q, et al. miR-484 regulates mitochondrial network through targeting Fis1. Nat Commun. 2012; 3: 781.

36. Liu $\mathrm{T}$, Wang $\mathrm{Z}$, Zhou R, Liang $\mathrm{W}$. Focally amplified lncRNA on chromosome 1 regulates apoptosis of esophageal cancer cells via DRP1 and mitochondrial dynamics. IUBMB Life. 2019; 71: 254-60.

37. Tian T, Lv X, Pan G, Lu Y, Chen W, He W, et al. Long Noncoding RNA MPRL Promotes Mitochondrial Fission and Cisplatin Chemosensitivity via Disruption of Pre-miRNA Processing. Clinical cancer research : an official journal of the American Association for Cancer Research. 2019; 25: 3673-88.

38. Sun F, Zhou Q, Pang X, Xu Y, Rao Z. Revealing various coupling of electron transfer and proton pumping in mitochondrial respiratory chain. Current opinion in structural biology. 2013; 23: 526-38.

39. Kim K, Jutooru I, Chadalapaka G, Johnson G, Frank J, Burghardt R, et al. HOTAIR is a negative prognostic factor and exhibits pro-oncogenic activity in pancreatic cancer. Oncogene. 2013; 32: 1616-25.

40. Zheng $\mathrm{P}$, Xiong $\mathrm{Q}, \mathrm{Wu} \mathrm{Y}$, Chen $\mathrm{Y}$, Chen $\mathrm{Z}$, Fleming J, et al Quantitative Proteomics Analysis Reveals Novel Insights into Mechanisms of Action of Long Noncoding RNA Hox Transcript Antisense Intergenic RNA (HOTAIR) in HeLa Cells. Molecular \& Cellular Proteomics. 2015; 14: 1447-63.

41. Gottschalk B, Klec C, Leitinger G, Bernhart E, Rost R, Bischof H, et al. MICU1 controls cristae junction and spatially anchors mitochondrial $\mathrm{Ca}(2+)$ uniporter complex. Nat Commun. 2019; 10: 3732.

42. Kong $\mathrm{L}$, Zhou $\mathrm{X}$, Wu Y, Wang $\mathrm{Y}$, Chen $\mathrm{L}$, Li P, et al. Targeting HOTAIR induces mitochondria related apoptosis and inhibits tumor growth in head and neck squamous cell carcinoma in vitro and in vivo. Current Molecular Medicine. 2015; 15: 952-60.

43. Goding CR. Targeting the IncRNA SAMMSON Reveals Metabolic Vulnerability in Melanoma. Cancer cell. 2016; 29: 619-21.

44. Leucci E, Vendramin R, Spinazzi M, Laurette P, Fiers M, Wouters J, et al. Melanoma addiction to the long non-coding RNA SAMMSON. Nature. 2016; 531: 518-22.

45. Noh JH, Kim KM, Abdelmohsen K, Yoon JH, Panda AC, Munk R, et al. HuR and GRSF1 modulate the nuclear export and mitochondrial localization of the IncRNA RMRP. Genes Dev. 2016; 30: 1224-39.

46. Clemson CM, Hutchinson JN, Sara SA, Ensminger AW, Fox AH, Chess A, et al. An Architectural Role for a Nuclear Noncoding RNA: NEAT1 RNA Is Essential for the Structure of Paraspeckles. Molecular cell. 2009; 33: 717-26.

47. Wang Y, Hu SB, Wang MR, Yao RW, Wu D, Yang L, et al. Genome-wide screening of NEAT1 regulators reveals cross-regulation between paraspeckles and mitochondria. Nat Cell Biol. 2018; 20: 1145-58.

48. Ilenia D'Errico, Lorena Salvatore, Stefania Murzilli, et al. Peroxisome proliferator-activated receptor-gamma coactivator 1-alpha (PGC1alpha) is a metabolic regulator of intestinal epithelial cell fate. Proc Natl Acad Sci U S A. 2011; 108: 6603-8.

49. Long J, Badal SS, Ye Z, Wang Y, Ayanga BA, Galvan DL, et al. Long noncoding RNA Tug1 regulates mitochondrial bioenergetics in diabetic nephropathy. The Journal of clinical investigation. 2016; 126: 4205-18.

50. Li $\mathrm{O}$, Imataka $\mathrm{H}$, Morino $\mathrm{S}$, et al. Eukaryotic translation initiation factor $4 \mathrm{AIII}$ (eIF4AIII) is functionally distinct from eIF4AI and eIF4AII. Mol Cell Biol. 1999; 19: 7336-46.

51. Ferraiuolo MA, Lee CS, Ler LW, et al. A nuclear translation-like factor eIF4AIII is recruited to the mRNA during splicing and functions in nonsense-mediated decay. Proc Natl Acad Sci U S A. 2004; 101: 4118-23. 
52. Li N, Zhan X, Zhan X. The IncRNA SNHG3 regulates energy metabolism of ovarian cancer by an analysis of mitochondrial proteomes. Gynecol Oncol. 2018; 150: 343-54.

53. Ros S, Floter J, Kaymak I, Da Costa C, Houddane A, Dubuis S, et al. 6-Phosphofructo-2-kinase/fructose-2,6-biphosphatase 4 is essential for p53-null cancer cells. Oncogene. 2017; 36: 3287-99.

54. Kawauchi K, Araki K, Tobiume K, Tanaka N. p53 regulates glucose metabolism through an IKK-NF-kappaB pathway and inhibits cell transformation. Nat Cell Biol. 2008; 10: 611-8.

55. Matoba S, Kang JG, Patino WD, Wragg A, Boehm M, Gavrilova O, et al. p53 regulates mitochondrial respiration. Science. 2006; 312: 1650-3.

56. Khan MR, Xiang S, Song Z, Wu M. The p53-inducible long noncoding RNA TRINGS protects cancer cells from necrosis under glucose starvation. EMBO J. 2017; 36: 3483-500.

57. Huarte M, Guttman M, Feldser D, Garber M, Koziol MJ, Kenzelmann-Broz D, et al. A large intergenic noncoding RNA induced by p53 mediates global gene repression in the p53 response. Cell. 2010; 142: 409-19.

58. Wang $\mathrm{X}, \mathrm{Xu} \mathrm{Y}$, Wang X, Jiang C, Han S, Dong K, et al. LincRNA-p21 suppresses development of human prostate cancer through inhibition of PKM2. Cell Prolif. 2017; 50 .

59. Yang F, Zhang H, Mei Y, Wu M. Reciprocal Regulation of HIF-1a and LincRNA-p21 Modulates the Warburg Effect. Molecular cell. 2014; 53: 88-100.

60. Dang CV, Le A, Gao P. MYC-Induced Cancer Cell Energy Metabolism and Therapeutic Opportunities. Clinical Cancer Research. 2009; 15: 6479-83.

61. Hung CL, Wang LY, Yu YL, Chen HW, Srivastava S, Petrovics G, et al. A long noncoding RNA connects c-Myc to tumor metabolism. Proceedings of the National Academy of Sciences of the United States of America. 2014; 111: 18697-702.

62. Feng J, Ma J, Liu S, Wang J, Chen Y. A noncoding RNA LINC00504 interacts with c-Myc to regulate tumor metabolism in colon cancer. Journal of cellular biochemistry. 2019.

63. Xiao ZD, Han L, Lee H, Zhuang L, Zhang Y, Baddour J, et al. Energy stress-induced lncRNA FILNC1 represses c-Myc-mediated energy metabolism and inhibits renal tumor development. Nat Commun. 2017; 8: 783.

64. Xue $\mathrm{M}, \mathrm{Li} \mathrm{X}, \mathrm{Li} \mathrm{Z}$, Chen $\mathrm{W}$. Urothelial carcinoma associated 1 is a hypoxia-inducible factor-1alpha-targeted long noncoding RNA that enhances hypoxic bladder cancer cell proliferation, migration, and invasion. Tumour biology : the journal of the International Society for Oncodevelopmental Biology and Medicine. 2014; 35: 6901-12.

65. Li Z, Li X, Wu S, Xue M, Chen W. Long non-coding RNA UCA1 promotes glycolysis by upregulating hexokinase 2 through the mTOR-STAT3/microRNA143 pathway. Cancer Sci. 2014; 105: 951-5.

66. Sun LY, Li XJ, Sun YM, Huang W, Fang K, Han C, et al. LncRNA ANRIL regulates AML development through modulating the glucose metabolism pathway of AdipoR1/AMPK/SIRT1. Molecular cancer. 2018; 17: 127.

67. Yang B, Zhang L, Cao Y, Chen S, Cao J, Wu D, et al. Overexpression of IncRNA IGFBP4-1 reprograms energy metabolism to promote lung cancer progression. Molecular cancer. 2017; 16: 154.

68. Rupaimoole R, Lee J, Haemmerle M, Ling H, Previs RA, Pradeep S, et al. Long Noncoding RNA Ceruloplasmin Promotes Cancer Growth by Altering Glycolysis. Cell reports. 2015; 13: 2395-402.

69. Sang LJ, Ju HQ, Liu GP, Tian T, Ma GL, Lu YX, et al. LncRNA CamK-A Regulates $\mathrm{Ca}(2+)$-Signaling-Mediated Tumor Microenvironment Remodeling. Molecular cell. 2018; 72: 71-83 e7.

70. Malakar P, Stein I, Saragovi A, Winkler R, Stern-Ginossar N, Berger M, et al. Long Noncoding RNA MALAT1 Regulates Cancer Glucose Metabolism by Enhancing mTOR-Mediated Translation of TCF7L2. Cancer research. 2019; 79: 2480-93.

71. Berteaux N, Lottin S, Monte D, Pinte S, Quatannens B, Coll J, et al. H19 mRNA-like noncoding RNA promotes breast cancer cell proliferation through positive control by E2F1. The Journal of biological chemistry. 2005; 280: 29625-36.

72. Liu C, Chen Z, Fang J, Xu A, Zhang W, Wang Z. H19-derived miR-675 contributes to bladder cancer cell proliferation by regulating p53 activation. Tumour biology : the journal of the International Society for Oncodevelopmental Biology and Medicine. 2016; 37: 263-70.

73. Shi Y, Wang Y, Luan W, Wang P, Tao T, Zhang J, et al. Long non-coding RNA H19 promotes glioma cell invasion by deriving miR-675. PLoS One. 2014; 9: e86295.

74. Lv J, Yu YQ, Li SQ, Luo L, Wang Q. Aflatoxin B1 promotes cell growth and invasion in hepatocellular carcinoma HepG2 cells through H19 and E2F1. Asian Pacific journal of cancer prevention : APJCP. 2014; 15: 2565-70.

75. Luan W, Zhou Z, Ni X, Xia Y, Wang J, Yan Y, et al. Long non-coding RNA H19 promotes glucose metabolism and cell growth in malignant melanoma via miR-106a-5p/E2F3 axis. J Cancer Res Clin Oncol. 2018; 144: 531-42.

76. Kang Y, Zhu X, Xu Y, Tang Q, Huang Z, Zhao Z, et al. Energy stress-induced IncRNA HAND2-AS1 represses HIF1alpha-mediated energy metabolism and inhibits osteosarcoma progression. Am J Cancer Res. 2018; 8: 526-37.

77. Song J, Wu X, Liu F, Li M, Sun Y, Wang Y, et al. Long non-coding RNA PVT1 promotes glycolysis and tumor progression by regulating miR-497/HK2 axis in osteosarcoma. Biochemical and biophysical research communications. 2017; 490: 217-24.

78. Chen J, Yu Y, Li H, Hu Q, Chen X, He Y, et al. Long non-coding RNA PVT1 promotes tumor progression by regulating the miR-143/HK2 axis in gallbladder cancer. Molecular cancer. 2019; 18: 33.
79. Xing Z, Zhang Y, Liang K, Yan L, Xiang Y, Li C, et al. Expression of Long Noncoding RNA YIYA Promotes Glycolysis in Breast Cancer. Cancer research. 2018; 78: 4524-32.

80. Xiang S, Gu H, Jin L, Thorne RF, Zhang XD, Wu M. LncRNA IDH1-AS1 links the functions of c-Myc and HIF1alpha via IDH1 to regulate the Warburg effect. Proc Natl Acad Sci U S A. 2018; 115: E1465-E74

81. Pan Y, Mansfield KD, Bertozzi CC, Rudenko V, Chan DA, Giaccia AJ, et al. Multiple Factors Affecting Cellular Redox Status and Energy Metabolism Modulate Hypoxia-Inducible Factor Prolyl Hydroxylase Activity In Vivo and In Vitro. Molecular and cellular biology. 2006; 27: 912-25.

82. Jaakkola P, Mole DR, Tian YM, Wilson MI, Gielbert J, Gaskell SI, et al. Targeting of HIF-alpha to the von Hippel-Lindau ubiquitylation complex by O2-regulated prolyl hydroxylation. Science. 2001; 292: 468-72.

83. Zheng YL, Li L, Jia YX, Zhang BZ, Li JC, Zhu YH, et al. LINC01554-Mediated Glucose Metabolism Reprogramming Suppresses Tumorigenicity in Hepatocellular Carcinoma via Downregulating PKM2 Expression and Inhibiting Akt/mTOR Signaling Pathway. Theranostics. 2019; 9: 796-810.

84. Bian Z, Zhang J, Li M, Feng Y, Wang X, Zhang J, et al. LncRNA-FEZF1-AS1 Promotes Tumor Proliferation and Metastasis in Colorectal Cancer by Regulating PKM2 Signaling. Clin Cancer Res. 2018; 24: 4808-19.

85. Banfai B, Jia H, Khatun J, Wood E, Risk B, Gundling WE, Jr., et al. Long noncoding RNAs are rarely translated in two human cell lines. Genome Res. 2012; 22: 1646-57.

86. Huang JZ, Chen M, Chen, Gao XC, Zhu S, Huang H, et al. A Peptide Encoded by a Putative IncRNA HOXB-AS3 Suppresses Colon Cancer Growth. Molecular cell. 2017; 68: 171-84 e6.

87. Patra KC, Hay N. The pentose phosphate pathway and cancer. Trends Biochem Sci. 2014; 39: 347-54.

88. Langbein S, Frederiks WM, zur Hausen A, Popa J, Lehmann J, Weiss C, et al. Metastasis is promoted by a bioenergetic switch: new targets for progressive renal cell cancer. Int J Cancer. 2008; 122: 2422-8.

89. Chen $Y, X u$ Q, Ji D, Wei Y, Chen H, Li T, et al. Inhibition of pentose phosphate pathway suppresses acute myelogenous leukemia. Tumour biology : the journal of the International Society for Oncodevelopmental Biology and Medicine. 2016; 37: 6027-34

90. Yang X, Ye H, He M, Zhou X, Sun N, Guo W, et al. LncRNA PDIA3P interacts with c-Myc to regulate cell proliferation via induction of pentose phosphate pathway in multiple myeloma. Biochemical and biophysical research communications. 2018; 498: 207-13.

91. DeBerardinis RJ, Cheng T. Q's next: the diverse functions of glutamine in metabolism, cell biology and cancer. Oncogene. 2010; 29: 313-24.

92. Locasale JW. Serine, glycine and one-carbon units: cancer metabolism in full circle. Nat Rev Cancer. 2013; 13: 572-83.

93. Tomlinson I, Webb E, Carvajal-Carmona L, Broderick P, Kemp Z, Spain S, et al. A genome-wide association scan of tag SNPs identifies a susceptibility variant for colorectal cancer at 8q24.21. Nature genetics. 2007; 39: 984-8.

94. Li J, Song P, Jiang T, Dai D, Wang H, Sun J, et al. Heat Shock Factor 1 Epigenetically Stimulates Glutaminase-1-Dependent mTOR Activation to Promote Colorectal Carcinogenesis. Mol Ther. 2018; 26: 1828-39.

95. Zhang J, Wang C, Chen M, Cao J, Zhong Y, Chen L, et al. Epigenetic silencing of glutaminase 2 in human liver and colon cancers. BMC Cancer. 2013; 13: 601.

96. D'Autreaux B, Toledano MB. ROS as signalling molecules: mechanisms that generate specificity in ROS homeostasis. Nat Rev Mol Cell Biol. 2007; 8: 813-24.

97. Li HJ, Li X, Pang H, Pan JJ, Xie XJ, Chen W. Long non-coding RNA UCA1 promotes glutamine metabolism by targeting miR-16 in human bladder cancer. Jpn J Clin Oncol. 2015; 45: 1055-63.

98. Deng SJ, Chen HY, Zeng Z, Deng S, Zhu S, Ye Z, et al. Nutrient Stress-Dysregulated Antisense IncRNA GLS-AS Impairs GLS-Mediated Metabolism and Represses Pancreatic Cancer Progression. Cancer research. 2019; 79: 1398-412

99. Wang P, Xu J, Wang Y, Cao X. An interferon-independent lncRNA promotes viral replication by modulating cellular metabolism. Science. 2017; 358: 1051-5.

100. Zeng B, Ye H, Chen J, Cheng D, Cai C, Chen G, et al. LncRNA TUG1 sponges miR-145 to promote cancer progression and regulate glutamine metabolism via Sirt3/GDH axis. Oncotarget. 2017; 8: 113650-61.

101. Liao M, Liao W, Xu N, Li B, Liu F, Zhang S, et al. LncRNA EPB41L4A-AS1 regulates glycolysis and glutaminolysis by mediating nucleolar translocation of HDAC2. EBioMedicine. 2019; 41: 200-13

102. Pascual G, Avgustinova A, Mejetta S, Martin M, Castellanos A, Attolini CS, et al. Targeting metastasis-initiating cells through the fatty acid receptor CD36. Nature. 2017; 541: 41-5.

103. Viswanathan VS, Ryan MJ, Dhruv HD, Gill S, Eichhoff OM, Seashore-Ludlow B, et al. Dependency of a therapy-resistant state of cancer cells on a lipid peroxidase pathway. Nature. 2017; 547: 453-7.

104. Jeon SM, Chandel NS, Hay N. AMPK regulates NADPH homeostasis to promote tumour cell survival during energy stress. Nature. 2012; 485: 661-5.

105. Rawson RB. Control of lipid metabolism by regulated intramembrane proteolysis of sterol regulatory element binding proteins (SREBPs). Biochemical Society symposium. 2003: 221-31.

106. Li D, Cheng M, Niu Y, Chi X, Liu X, Fan J, et al. Identification of a novel human long non-coding RNA that regulates hepatic lipid metabolism by inhibiting SREBP-1c. Int J Biol Sci. 2017; 13: 349-57.

107. Peters JM, Shah YM, Gonzalez FJ. The role of peroxisome proliferator-activated receptors in carcinogenesis and chemoprevention. Nat Rev Cancer. 2012; 12: 181-95. 
108. Liu X, Liang Y, Song R, Yang G, Han J, Lan Y, et al. Long non-coding RNA NEAT1-modulated abnormal lipolysis via ATGL drives hepatocellular carcinoma proliferation. Molecular cancer. 2018; 17: 90.

109. Adriaens C, Standaert L, Barra J, Latil M, Verfaillie A, Kalev P, et al. p53 induces formation of NEAT1 IncRNA-containing paraspeckles that modulate replication stress response and chemosensitivity. Nat Med. 2016; 22: 861-8.

110. Mello SS, Sinow C, Raj N, Mazur PK, Bieging-Rolett K, Broz DK, et al. Neat1 is a p53-inducible lincRNA essential for transformation suppression. Genes Dev. 2017; 31: 1095-108

111. Zhang X, Xue C, Lin J, Ferguson JF, Weiner A, Liu W, et al. Interrogation of nonconserved human adipose lincRNAs identifies a regulatory role of linc-ADAL in adipocyte metabolism. Science translational medicine. 2018; 10.

112. Cui M, Xiao Z, Wang Y, Zheng M, Song T, Cai X, et al. Long noncoding RNA HULC modulates abnormal lipid metabolism in hepatoma cells through an miR-9-mediated RXRA signaling pathway. Cancer research. 2015; 75: 846-57.

113. Shang C, Wang W, Liao Y, Chen Y, Liu T, Du Q, et al. LNMICC Promotes Nodal Metastasis of Cervical Cancer by Reprogramming Fatty Acid Metabolism. Cancer research. 2018; 78: 877-90.

114. Kogure T, Yan IK, Lin WL, Patel T. Extracellular Vesicle-Mediated Transfer of a Novel Long Noncoding RNA TUC339: A Mechanism of Intercellular Signaling in Human Hepatocellular Cancer. Genes \& cancer. 2013; 4: 261-72.

115. Chen F, Chen J, Yang L, Liu J, Zhang X, Zhang Y, et al. Extracellular vesicle-packaged HIF-1alpha-stabilizing lncRNA from tumour-associated macrophages regulates aerobic glycolysis of breast cancer cells. Nature cell biology. 2019; 21: 498-510.

116. Marsh T, Pietras K, McAllister SS. Fibroblasts as architects of cancer pathogenesis. Biochim Biophys Acta. 2013; 1832: 1070-8.

117. Zhao L, Ji G, Le X, Wang C, Xu L, Feng M, et al. Long Noncoding RNA LINC00092 Acts in Cancer-Associated Fibroblasts to Drive Glycolysis and Progression of Ovarian Cancer. Cancer research. 2017; 77: 1369-82.

118. He Z, You C, Zhao D. Long non-coding RNA UCA1/miR-182/PFKFB2 axis modulates glioblastoma-associated stromal cells-mediated glycolysis and invasion of glioma cells. Biochemical and biophysical research communications. 2018; 500: 569-76.

119. Ding L, Ren J, Zhang D, Li Y, Huang X, Hu Q, et al. A novel stromal lncRNA signature reprograms fibroblasts to promote the growth of oral squamous cell carcinoma via LncRNA-CAF/interleukin-33. Carcinogenesis. 2018; 39: 397-406.

120. Xiao P, Wan X, Cui B, Liu Y, Qiu C, Rong J, et al. Interleukin 33 in tumor microenvironment is crucial for the accumulation and function of myeloid-derived suppressor cells. Oncoimmunology. 2016; 5: e1063772.

121. Leucci E, Vendramin R, Spinazzi M, Laurette P, Fiers M, Wouters J, et al. Melanoma addiction to the long non-coding RNA SAMMSON. Nature. 2016; 531: 518-+.

122. Alessio E, Buson L, Chemello F, Peggion C, Grespi F, Martini P, et al. Single cell analysis reveals the involvement of the long non-coding RNA Pvt1 in the modulation of muscle atrophy and mitochondrial network. Nucleic Acids Res. 2019; 47: 1653-70. 